\title{
Can Understanding Outcomes for Medicare Advantage Enrollees Reduce Racial and Ethnic Disparities in Kidney Disease?
}

\author{
Keith C. Norris \\ Division of General Internal Medicine and Health Services Research, Department of Medicine, \\ University of California, Los Angeles, CA, USA
}

It is well established that the burden of chronic kidney disease (CKD) falls disproportionately upon members of racial and ethnic minority communities driven mainly by higher rates of major CKD risk factors such as diabetes, hypertension, lower socioeconomic status, and limited access to care [1]. A recent Kidney Disease: Improving Global Outcomes (KDIGO) controversies conference on "Early Identification and Intervention in CKD" found that many available guidelines on evaluating and managing early CKD have not been universally adopted, leading them to recommend immediate implementation of CKD screening coupled with risk stratification and treatment for high-risk persons, preferably in primary or community care settings [1]. Identification of traditional and novel CKD progression factors may enable providers to better provide high-value clinical care, a major goal for the nephrology and primary care communities.

While several strategies such as KDIGO guidelines are critical to our present approaches to CKD care, the use of different large data sets can provide additional insights into CKD risk and protective factors that can help refine and possibly promote the harmonization of prediction models for CKD progression and/or complications, and help identify, validate, and refine stratification of highrisk populations in different settings [2, 3]. For instance, an observational analysis of data from nearly 700,000 veterans with a mean age of 73 years with stage 3 or 4 CKD followed up for over a nearly 6-year period found that both Black and Hispanic veterans experienced faster progression to CKD stage 5 than White veterans despite having higher rates of nephrology referral and visits, suggesting other factors are at play [3]. Given the veteran's population is $90 \%$ male and other factors that may lead to military service-level selection bias, it is important to examine CKD progression in other large diverse cohorts that may yield even more generalizable information.

One such large population at high risk for CKD that is highly representative of a large portion of older Americans are persons covered by Medicare, a form of federal government health insurance for persons over 65 years of age or disabled (including persons with end-stage kidney disease). Traditionally, Medicare patients have received treatment in fee-for-service (FFS) health-care models. However, over the last 15 years, there has been a progressive increase in the percent of Medicare recipients receiving their benefits through Medicare Advantage plans, a 
form of health insurance that provides Medicare benefits through a private-sector health insurer [4]. As of 2020, there were $>24$ million Medicare beneficiaries enrolled in Medicare Advantage plans, representing nearly $40 \%$ of the Medicare population (and 50\% of the Black and Hispanic Medicare population), making it an important population to understand $[4,5]$.

Reports of outcomes for patients in traditional FFS compared to Medicare Advantage have shown variable results. Frean et al. [6] found no difference in 8 previously established domains of patient-perceived integrated care between patients enrolled in Medicare Advantage and traditional Medicare FFS plans. By contrast, Welch et al. [7] reported greater physician concentration of services across Medicare advantage insurers than FFS was associated with a higher quality of care overall and especially among vulnerable enrollees. Medicare Advantage enrollees also appear to have more preventive services and less out-of-pocket costs [5]. Thus, Medicare Advantage participants are a rapidly increasing group of insured Americans that, while similar, may also have some unique characteristics in comparison to their Medicare FFS peers.

To this end, in this issue of $A J N$, Clarissa Diamantidis et al. [8] present the first study to examine CKD progression in a large longitudinal cohort of Medicare Advantage enrollees followed up for over 3 years, with over $96 \%$ having stage 2 or $3 \mathrm{CKD}$. They found that Black and Hispanic Medicare Advantage enrollees had faster kidney function decline over time than their White peers, even after adjusting for comorbidities and despite having greater kidney function at study entry, consistent with the work of Suarez et al. [3].

This is particularly important for patients with stage 2 or $3 \mathrm{CKD}$ where primary care providers are most likely to start paying closer attention to $\mathrm{CKD}$-specific guidelines and are likely early enough to be able to have the greatest impact on preventing disease progression and complications $[1,8]$. They also found that Medicare Advantage enrollees with clinically recognized CKD had both lower estimated glomerular filtration rate (eGFR) levels and slower decline in kidney function than patients without clinically recognized CKD [8]. This could be due to patients with clinical recognition of CKD having received closer monitoring and increased receipt of nephrology consultation, which may have led to more aggressive care or other. Diamantidis et al. [8] also found removal of the eGFR race coefficient in Black enrollees led to a nearly 10 $\mathrm{mL} / \mathrm{min} / 1.73 \mathrm{~m}^{2}$ reduction in baseline eGFR, suggesting the use of a race modifier could mask timely identifica-

Medicare Advantage Plans and Kidney Disease Disparities tion and clinical management of CKD among Black enrollees. It is important to note the higher initial eGFR estimates that are associated with an accelerated decline in kidney function over time may be reflective of hyperfiltration, which may be a reflection of increased dietary protein intake, elevated blood pressure, hyperglycemia, medications, and more which may conspire to mask the risk for future decline in kidney function $[9,10]$. Their findings reinforce the importance of assessing a profile of predicted risk, rather than the too often used single eGFR alone.

Important study limitations include the high number of missing serum creatinine, or urinary albumin-to-creatinine lab values $(>3.4 \mathrm{M})$ and lack of 2 serum creatinine values at least 90 days apart indicating actual CKD, although sensitivity analyses suggested these patient cohorts had a somewhat similar demographic clinical profile. Also, the lack of data on medications, especially angiotensin-converting enzyme inhibitor or angiotensin receptor blocker use and their relation with eGFR progression, as well as if medication use differed between groups could account, in part, for the finding. Another important consideration from the perspective of identifying high populations is the method of ascertainment of race and ethnicity, which were derived from a national supplier of consumer marketing data using a proprietary methodology and then imputed. Whether this imputation approximates individual level identity or residence in neighborhoods using addresses to estimate prevalence rates of different racial groups at a zip code or census track level is not clear and can have important implications on the interpretation of the role of race and ethnicity. It is also important to recognize that in research race and ethnicity should be clearly defined and even then used carefully, possibly as a contextual variable in a regression and not as a biologic outcome variable to generate an individual level race or ethnicity modifier [11]. Race and ethnicity are conceptual social terms that can provide group level estimates, but neither can be consistently measured nor can they inform us what their indirect association with a health condition or outcome means at an individual level $[11,12]$. Race and ethnicity capture a gross estimate of how a group is treated, its social class and culture in a race-stratified society as well as gene polymorphism prevalence, biomarkers, and more that vary tremendously across each individual within and across racial and ethnic groups [11, 12]. Thus, prediction models are limited to only being able to estimate associations with race and ethnicity at a group level, but this can be valuable to inform population level actions 
such as targeted population level education, outreach, and messaging and designing group level interventions and monitoring outcomes. With a thoughtful approach to identifying groups at higher risk for CKD progression and complications, we can advance health equity and improve outcomes for all of the patients who trust us to provide the highest quality of care and excellence and compassion.

\section{Conflict of Interest Statement}

There is no conflict of interest.

\section{Funding Sources}

K.N. is supported in part by NIH Grants UL1TR000124 and P30AG021684.

\section{References}

1 Shlipak MG, Tummalapalli SL, Boulware LE, Grams ME, Ix JH, Jha V, et al. The case for early identification and intervention of chronic kidney disease: conclusions from a kidney disease: improving global outcomes (KDIGO) controversies conference. Kidney Int. 2021;99:34-47.

2 Tangri N, Stevens LA, Griffith J, Tighiouart $\mathrm{H}$, Djurdjev O, Naimark D, et al. A predictive model for progression of chronic kidney disease to kidney failure. JAMA. 2011;305:15539.

3 Suarez J, Cohen JB, Potluri V, Yang W, Kaplan DE, Serper M, et al. Racial disparities in nephrology consultation and disease progression among veterans with CKD: an observational cohort study. J Am Soc Nephrol. 2018; 29:2563-73.

4 Jacobson G, Damico A, Neuman T. A dozen facts about medicare advantage. Washington: Kaiser Family Foundation; 2021.
5 Medicare advantage offers high quality care and cost protections to racially and ethnically diverse beneficiaries. Data Brief. June 2021. Accessed 2021 Feb 9. Available from: https:// bettermedicarealliance.org/wp-content/uploads/2021/06/BMA_2021-Q2-DataBrief_6.15.21.pdf.

6 Frean M, Covington C, Tietschert M, Bahadurzada H, So J, Singer SJ. Patient experiences of integrated care in medicare Accountable Care Organizations and medicare advantage versus traditional fee-for-service. Med Care. 2021;59(3):195-201.

7 Welch WP, Sen AP, Bindman AB. Concentration of physician services across insurers and effects on quality: early evidence from medicare advantage. Med Care. 2019;57:795-800.
8 Diamantidis CJ, Zepel L, Wang V, Smith VA, Scholle SH, Tamayo L, et al. Disparities in chronic kidney disease progression by medicare advantage enrollees. Am J Nephrol. doi: $10.1159 / 000519758$. Epub ahead of print.

9 Rossitto G, Maiolino G, Lerco S, Ceolotto G, Blackburn G, Mary S, et al. High sodium intake, glomerular hyperfiltration, and protein catabolism in patients with essential hypertension. Cardiovasc Res. 2021;117(5):137281.

10 Tuttle KR. Back to the future: glomerular hyperfiltration and the diabetic kidney. Diabetes. 2017;66:14-6.

11 Jones CP. Invited commentary: "race," racism, and the practice of epidemiology. Am J Epidemiol. 2001; 154:299-304; discussion 305-6.

12 Robinson WS. Ecological correlations and the behavior of individuals. Am Sociol Rev. 1950; 15(3):351-7. 